\title{
Plastome of mycoheterotrophic Burmannia itoana Mak. (Burmanniaceae) exhibits extensive degradation and distinct rearrangements
}

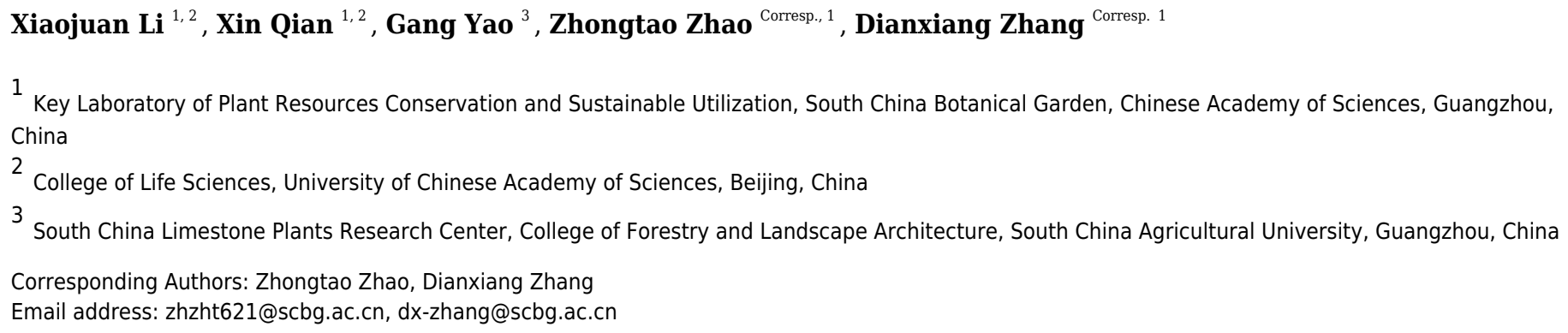

Plastomes of heterotrophs went through varying degrees of degradation along with the transition from autotrophic to heterotrophic lifestyle. Here we identified the plastome of mycoheterotrophic species Burmannia itoana and compared it with those of its reported relatives including three autotrophs and one heterotroph (Thismia tentaculata) in Dioscoreales. $B$. itoana yields a rampantly degraded plastome reduced in size and gene numbers at the advanced stages of degradation. Its length is $44,463 \mathrm{bp}$ with a quadripartite structure. $B$. itoana plastome contains 33 tentatively functional genes and six tentative pseudogenes, including several unusually retained genes. These unusual retention suggest that the inverted repeats regions (IRs) and possibility of being compensated may prolong retention of genes in plastome at the advanced stage of degradation. Otherwise, six rearrangements including four inversions (Inv1/Inv2/Inv3/Inv4) and two translocations (Trans1/Trans2) were detected in $B$. itoana plastome versus its autotrophic relative Burmannia disticha. We speculate that Inv1 may be mediated by recombination of distinct tRNA genes, while Inv2 is likely consequence of extreme gene losses due to the shift to heterotrophic lifestyle. The other four rearrangements involved in IRs and SSC may attribute to multiple waves of IRs and overlapping inversions. Our study fills the gap of knowledge about plastomes of heterotroph in Burmannia and provides a new evidence for the convergent degradation patterns of plastomes en route to heterotrophic lifestyle. 


\section{Plastome of mycoheterotrophic Burmannia itoana}

2 Mak. (Burmanniaceae) exhibits extensive degradation

3 and distinct rearrangements

4

Xiaojuan $\mathrm{Li}^{1,2}$, Xin Qian ${ }^{1,2}$, Gang $\mathrm{Yao}^{3}$, Zhongtao Zhao ${ }^{1}$, Dianxiang Zhang ${ }^{1}$

${ }^{1}$ Key Laboratory of Plant Resources Conservation and Sustainable Utilization, South China

8 Botanical Garden, Chinese Academy of Sciences, Guangzhou, China

$9{ }^{2}$ College of Life Sciences, University of Chinese Academy of Sciences, Beijing, China

$10{ }^{3}$ South China Limestone Plants Research Center, College of Forestry and Landscape

11 Architecture, South China Agricultural University, Guangzhou, China

Corresponding Author:

Zhongtao Zhao ${ }^{1}$, Dianxiang Zhang ${ }^{1}$

Email address: zhzht621@scbg.ac.cn; dx-zhang@scbg.ac.cn 
36

37

38

39

40

41

42

43

44

45

46

47

48

49

50

51

52

53

54

55

56

57

58

59

60

61

62

63

64

65

66

67

68

69

70

71

72

73

74

75

\section{Abstract}

Plastomes of heterotrophs went through varying degrees of degradation along with the transition from autotrophic to heterotrophic lifestyle. Here we identified the plastome of mycoheterotrophic species Burmannia itoana and compared it with those of its reported relatives including three autotrophs and one heterotroph (Thismia tentaculata) in Dioscoreales. B. itoana yields a rampantly degraded plastome reduced in size and gene numbers at the advanced stages of degradation. Its length is 44,463 bp with a quadripartite structure. $B$. itoana plastome contains 33 tentatively functional genes and six tentative pseudogenes, including several unusually retained genes. These unusual retention suggest that the inverted repeats regions (IRs) and possibility of being compensated may prolong retention of genes in plastome at the advanced stage of degradation. Otherwise, six rearrangements including four inversions (Inv1/Inv2/Inv3/Inv4) and two translocations (Trans1/Trans2) were detected in B. itoana plastome versus its autotrophic relative Burmannia disticha. We speculate that Inv1 may be mediated by recombination of distinct tRNA genes, while Inv2 is likely consequence of extreme gene losses due to the shift to heterotrophic lifestyle. The other four rearrangements involved in IRs and SSC may attribute to multiple waves of IRs and overlapping inversions. Our study fills the gap of knowledge about plastomes of heterotroph in Burmannia and provides a new evidence for the convergent degradation patterns of plastomes en route to heterotrophic lifestyle.

\section{Introduction}

Plastids were derived from a common cyanobacterial ancestor that established a permanent endosymbiotic relationship with mitochondriate ancestor (Gould et al. 2008; Ku et al. 2015). Over the evolutionary history, the plastid encoding genes have undergone multiple transfers and losses which are ongoing processes. For instance, the ancestor genes of the plastid have transferred to other two genomes (nuclear and mitochondria) or total lost during evolution (Martin and Herrmann 1998; Cusimano and Wicke 2016). Most plastomes of land plants are canonical circular and quadripartite including two inverted repeats (IRs) separated by larger single copy region (LSC) and small single copy region (SSC). Most typical plastomes are variable in size ranging from approximate $120 \mathrm{kbp}$ to $170 \mathrm{kbp}$ with about 113 unique genes including about 79 protein-coding genes, four rRNA genes, and 30 tRNA genes (Wicke et al. 2011). These genes are classified into three main classes depending on functions: (1) photosynthesis related genes (ndh/atp/psa/psb/pet/ycf3/ycf4/rbcL); (2) transcription, transcript maturation and translation related genes (rpo/infA, matK and tRNAs/rRNAs/rps/rpl), (3) other non-bioenergetic function genes (accD/clpP/ycf1/ycf2/ccsA/cemA) (Bock 2007; Wicke et al. 2011).

Parasitic and mycoheterotrophic plants, which establish a physiological relationship with either plants or fungi to obtain organic and mineral nutrients, are referred to have partially or fully lost the capacity of photosynthesis (Wicke and Naumann 2018). The transition of lifestyle from autotrophic to heterotrophic may have left measurable clues in genomes, such as adaptive and non-adaptive changes (Wicke et al. 2016). Studies about plastomes of heterotrophs revealed that plastomes of heterotrophic plants experienced convergent degradation syndromes compared 
with those of autotrophs, such as overall decreases in genome size along with functional and physical gene losses, decrease in GC-content, increased frequency of rearrangements, accumulation of indels and losses of introns (Wicke et al. 2013; Logacheva et al. 2014; Lam et al. 2015; Lim et al. 2016; Wicke et al. 2016; Petersen et al. 2018; Schneider et al. 2018).

Hitherto, several conceptual models of plastome degradation have been proposed to account for the order of the heterotrophs plastomes degradation in a simplified and idealized manner. In general, plastome degradation follows five major stages (Barrett and Davis 2012; Barrett et al. 2014; Naumann et al. 2016; Wicke et al. 2016; Graham et al. 2017): (1) ndh genes will be affected first and functionally lost from the plastomes once plants have the ability of obtaining nutrients using heterotrophic way; (2) when the lifestyle transfer to obligate heterotrophic, most of photosynthesis related genes and some housekeeping genes are lost; (3) atp/rbcL and nonessential housekeeping genes are lost or functionally replaced; (4) other nonbioenergetic genes (e. g. accD, clpP, ycf1/2) are lost or functionally replaced; (5) all plastid encoding genes are lost.

Burmannia contains about 60 species spanning from autotrophic, hemi-mycoheterotrophic to mycoheterotrophic (Jonker 1938; Zhang 1999; Wu et al. 2010), which could provide an excellent model system for understanding the evolution of plastome responding to the lifestyle shift from autotrophic to heterotrophic. Although the plastome of autotrophic Burmannia disticha (Ma et al. 2018) has been described, the plastome of mycoheterotrophic species in Burmannia has never been reported, hindering our efforts in elucidation of plastid evolution in the genus. Aiming to provide new evidence in our understanding of the mechanism of plastid evolution in mycoheterotrophic angiosperms, here we focus on Burmannia itoana, a mycoheterotrophic perennial herb (Fig. 1) distributed in the coastal provinces of southern China and Ryukyu Islands of Japan (Jonker 1938; Zhang 1999; Wu et al. 2010). We sequenced the $B$. itoana plastome and examined its content and structure. Furthermore, we compared the plastome of B. itoana with those of its four documented relatives (B. disticha, Thismia tentaculata (Thismiaceae), Tacca chantrieri (Taccaceae), and Dioscorea zingiberensis (Dioscoreaceae)) in Dioscoreales.

\section{Materials and methods}

\section{DNA extraction and sequencing}

Samples of B. itoana were collected from the Longmen Park, Guangdong Province, China. The voucher specimens (LXJLM07) are deposited in the IBSC (Herbarium of South China Botanical Garden). Total DNA was extracted from an individual plant with an identical collection number as that of voucher specimens using a DNeasy Plant Mini Kit (Qiagen, Hilden, Germany). The total DNA was used to generate libraries with average insert size of $500 \mathrm{bp}$ and sequenced using Illumina HiSeq 2000 with 150 bp paired-end read lengths.

\section{Plastome de novo assembly and annotation}

The plastome was assembled using CLC Genomics Workbench v9.0 (CLC BIO, Aarhus, Denmark) with parameters as follows: wordsize 63, bubble size 50, minimal contig length 1000 bp. Three plastome-like contigs were picked out through mapping all assembled contigs to the 
116 sequence of $B$. disticha plastome, and then these contigs were merged using Geneious (version 117 11.1.5) (Kearse et al. 2012) to build draft plastomes. Specific primers were designed to confirm 118 the overlap of the aligned contigs and identify the borders of the LSC, SSC, and IRs regions 119 using through PCR and Sanger sequencing method with these primers as follows. LSC/IRa-F (5' 120 TGA GAC CTA GTG CTC AAG GGA 3'), LSC/IRa-R (5' AAG GTT TAA GAT TTG TAT 121 TTG AAA GA 3'); LSC/IRb-F (5' CGA GTC ACA CAC TAA GCA TAG C 3'), LSC/IRb-R 122 (5' TGA GAC CTA GTG CTC AAG GGA 3'); SSC/IRa-F (5' TCT CTT TAA CAT TTA TGA 123 CAC GAC A 3'), SSC/IRa-R (5' TTG CGA ACA TAC TCC CCA GG 3'); SSC/IRb-F (5' AAC GCG TTA GCT ACA GCA CT 3'), SSC/IRb-R (5' TGA GTT AGT GTG AGC TTA TCC 3'). Validated complete plastome was annotated using GeSeq (Tillich et al. 2017) with default sets. Furthermore, tRNAs were predicted using tRNAscan-SE (Schattner et al. 2005). Finally, the plastome map was visualized using OGDRAW v. 1.2 (Lohse et al. 2007). The final annotated plastome was deposited in GenBank under accession number MK318822, and annotation of plastome is summarized in Additional file 1. tentaculata (Thismiaceae), Tacca chantrieri (Taccaceae), and Dioscorea zingiberensis (Dioscoreaceae) were downloaded from GenBank, and the Genbank accession is MG792012, KX171421, KX171420 and NC_027090M, respectively.

The GC-content of plastome and the four junctions of LSC/IRB, LSC/IRA, SSC/IRB and SSC/IRA were identified using Geneious version 11.1.5 (Kearse et al. 2012). Gene contents of plastomes were compared between B. itoana and four reported species in Dioscoreales. Gene orders excluding copies in IRs were explored using Mauve (Darling et al. 2004) plugged in Geneious.

\section{Results}

\section{General characteristics of $B$. itoana plastome}

B. itoana plastome represents a quadripartite circular molecule containing two larger inverted repeat regions (IRA/IRB: 12,174 bp) separated by a large single-copy region (LSC: 18,441 bp) and a small single-copy region (SSC: 1674 bp) (Fig. 2). The B. itoana plastome is $44,636 \mathrm{bp}$ in length with 39 genes (Fig. 2, Fig. 3) including 33 putatively functional genes and six putative pseudogenes. The presumably functional genes include four rRNA genes, eight tRNA genes and 21 protein genes, while six genes (petG, rpl36, trnH_GUG, trnD_GUC, trnG_GCC and trmS_UGA) are identified as putative pseudogenes based on the reasons below: the presence of internal stop codons in petG and rpl36; the anticodon sequences of the trnH_GUG was deleted compared with the typical trnH_GUG; multiple base changes in anticodon and other regions in trnD_GUC compared with the functional trnD_GUC, and no tRNA was predicted by tRNAscan; several indels or substitutes in the trnG_GCC compared with the typical trnG_GCC, and no tRNA was predicted by tRNAscan; several indels or substitutes in the anticodon and other regions of the trmS_UGA compared with the typical trmS_UGA, and no tRNA was predicted by 
156 absent), while the clpP gene harbors two introns. The overall GC-content of $B$. itoana plastome 157 is $32 \%$, which is lower than those of its autotrophic relatives but is higher than that of $T$.

158 tentaculata. The GC-content of IR, LSC and SSC in B. itoana is $37.8 \%, 24.5 \%$ and $32.4 \%$, 159 respectively (Table 1 ).

160 Comparative analyses of gene contents and gene order

161 Each plastome of the autotrophic relatives $B$. disticha, T. chantrieri and D. zingiberensis contains 162112 genes (Fig. 3). The second intron of rps12 is lost in B. itoana plastome, whereas rps12 is 163 totally absent from the $T$. tentaculata plastome, while this gene is retained in each plastome of 164 the autotrophic relatives. Like plastome of T. tentaculata, B. itoana plastome exhibits not only 165 extensive reduction in length but also losses of most photosynthesis related genes and some 166 housekeeping genes, while only one photosynthesis related gene, named petG, is retained as a

167

168

169

170

171

172

173

174

175

176

177

178

179

180

181

182

183

184

185

186

187

188

189

190

191

192

193

194

195 pseudogene in B. itoana plastome. In addition, B. itoana plastome shares all of the 12 genes retained in $T$. tentaculata plastome. Compared with $B$. itoana plastome, $T$. tentaculata plastome has experienced further degradation.

Alignment using Mauve showed that plastomes of autotrophs are highly colinear with exceptions that the inversion of the SSC in B. disticha, and extremely modified plastome of $T$. tentaculata (Fig. 4). Here we mainly focus on the comparison between the plastome structure of B. itoana and B. disticha represented in Fig. 2 and Fig. 4. The results showed that gene order of B. itoana plastome exhibits six rearrangements including four inversions (Inv1, Inv2, Inv3, Inv4) and two translocations (Trans1/Trans2) versus that of $B$. disticha plastome. Inv1 contains the cluster of trnS_GUA - trnG_GCC - trnfM_CAU - rps14 (ca. 1282 bp), and Inv2 just contains accD (ca. 2725 bp), both of which are located in LSC. Inv3 contains the cluster of rps7 - rps 12 rrn16 -rrn23 - rrn4.5 - rrn5 (ca. 6678 bp) stretching across the IRs and SSC, and Inv4 contains the cluster of rrn16 - rrn23 - rrn4.5 - rrn5 (ca. 5007 bp) located in IRs. Trans1/Trans2 contain the cluster of partial truncate ndhD - rpl32 - rps15 - truncate ycf1 - trnN (ca. 2600 bp), andTrans1/Trans2 are involved in the IRs and SSC of $B$. disticha plastome.

\section{Boundaries of SC/IRs of Dioscoreales plastomes}

The boundary of LSC/IRB in B. itoana plastome slightly expands to rps3, which is different from that in its autotrophic relatives. Specifically, boundary of LSC/IRB in B. disticha is located in rpl22, and those of $T$. chantrieri and $D$. zingiberensis are located in the intergenic space between rps19 and trnH_GUG. The boundary of SSC/IR in B. itoana plastome resides in rps7. In contrasting to that, the boundaries of SSC/IR are located in the intergenic of rps15 and ycf1, ycf1 in B. disticha and D. zingiberensis, respectively. In T. chantrieri, one boundary of SSC/IRs expands to the intron of ndhA. In summary, IRs have undergone expansions in both plastomes of B. itoana and B. disticha relative to those in other compared lineages. The boundaries of SC/IRs of Dioscoreales plastomes are showed in Fig. 2.

\section{Discussion}

\section{Convergent evolution of plastomes in heterotrophs}

Accompanying with forfeiting of photosynthetic capabilities, the plastomes of heterotrophic plants undergo varying degrees of degradation in size and gene contents (Wicke et al. 2013; 
196

197

198

199

200

201

202

203

204

205

206

207

208

209

210

211

212

213

214

215

216

217

218

219

220

221

222

223

224

225

226

227

228

229

230

231

232

233

234

235

Wicke et al. 2016). For instance, the largest plastome is that of heterotrophic chlamydomonadalean alga Polytoma uvella with about $230 \mathrm{~kb}$ in length (Figueroa-Martinez et al. 2017), while the smallest sequenced plastome is 11,348 bp from endoparastic Pilostyles aethiopica (Bellot and Renner 2015). Moreover, previous studies reported that plastome has possibly been totally lost in green algal genus Polytomella (Smith and Lee 2014) and land flowering plant Rafflesia lagascae (Molina et al. 2014). Our results show that B. itoana plastome displays a typical quadripartite architecture with LSC and SSC separated by IRs consistent with that of canonical land plant plastomes. The length of $B$. itoana platome is only one-third of its autotrophic relative $B$. distich $a$ and is nearly three times that of heterotrophic T. tentaculata. As previously mentioned, gene losses in plastomes of heterotrophs follow five stages (Barrett and Davis 2012; Barrett et al. 2014; Naumann et al. 2016; Graham et al. 2017; Wicke and Naumann 2018). Accordingly, B. itoana plastome is likely at the fourth stage with non-bioenergetic and housekeeping genes (e. g., ycf1/2, rps/rpl/tRNAs) being physically or functionally lost. Losses of ycf1 and ycf2 in B. itoana plastome support that a general core set of genes (ycf1, ycf2, accD, clpP, infA and trnE) varies among heterotrophs (Bellot and Renner 2015; Schelkunov et al. 2015; Lim et al. 2016; Naumann et al. 2016; Roquet et al. 2016).

It is a well-accepted tenet that matK encodes one kind of protein required for group IIA intron splicing of seven plastid genes trnV_UAC, trnI_GAU, trnK_UUU, $\operatorname{trn} A \_U G C, \operatorname{rp} 12, \operatorname{rps} 12$ (3-end intron) and atpF (Liere and Link 1995; Zoschke et al. 2010). Normally, the group IIA intron will not be spliced out from the transcripts without matK. Parallel losses of matK and group IIA intron-containing genes have been reported in Cuscuta (McNeal et al. 2009). In B. itoana plastome, matK is physically lost accompanying with the loss of other six genes containg group IIA intron, however, rpl2 and rps12 (the second intron of rps 12 is lost) are retained. Similarly, matK is absent or truncated in the plastomes of heterotrophic Cynomorium coccineum, Rhizanthella gardneri and Epipogium aphyllum, while the second intron of rps12 is lost but rpl2 remains intact (Delannoy et al. 2011; Bellot and Renner 2015; Schelkunov et al. 2015). However, the introns of both rpl2 and rps 12 are still sustained while matK are absent in Hydnora visseri plastome, and all rps12 and rpl2 are transcribed properly (Naumann et al. 2016). In addition, a large proportion of matK in some species of orchids are pseudogenes while all of the seven genes with group II introns are retained (Kim et al. 2014; Feng et al. 2016). These instances of matK loss or pseudogenization with retention of group IIA introns may be because that the function of matK could be recovered by that of genes from nucleus (Mohr and Lambowitz 2003), or some group IIA introns may be able to self-splice (Kim et al. 2014).

Most reported plastomes at the advanced stage of degradation have experienced multiple losses of tRNA genes reviewed in Graham et al (2017) and Wicke \& Naumann (2018), while plastomes of autotrophs typically harbor 30 tRNAs. Combinations of reported plastomes exhibit that the number of tRNAs retained in plastomes at the advanced stage of degradation ranges from zero in two endoparasitic species of Pilostyles (Bellot and Renner 2015) to 24 in Orobanche gracilis (Genbank accession number: NC_023464, Orobanchaceae). Without exception, only 12 tRNAs (eight functional and four pseudogenized) are retained in the B. itoana 
236 plastome. In addition, comparative analyses show that most plastomes contain a unique set of 237 tRNAs in heterotrophs. For instance, the subset of tRNAs remained in B. itoana plastome has not

238

239

240

241

242

243

244

245

246

247

248

249

250

251

252

253

254

255

256

257

258

259

260

261

262

263

264

265

266

267

268

269

270

271

272

273

274

275

been detected in others. It shares only eight tRNAs with $R$. gardneri (containing 10 tRNAs) (Delannoy et al. 2011), seven with E. roseum (containing eight tRNAs) (Schelkunov et al. 2015) and six with Sciaphila densiflora (containing six tRNAs) (Lam et al. 2015). Moreover, a question is raised that tRNAs remained in plastomes of heterotrophs are due to adaptive selection or have just fortuitously escaped deletion. Computer simulations suggest that about half of tRNAs in plastome of Epifagus virginiana are retained by chance and half are maintained by selection (Lohan and Wolfe 1998). It was a plausible explanation that the two tRNAs (trnE_UUC and trnfM_CAU) are essential for haem biosynthesis and mitochondrial protein synthesis, respectively, and cannot be replaced with their cytosolic orthologues (Barbrook et al. 2006). Previous study showed that two tRNAs (trnE_UUC and trnfM_CAU) are shared within most heterotrophs. Without exception, B. itoana plastome shares two tRNAs (trnE_UUC and trnfM_CAU) with those of T. tentaculata (Merckx et al. 2017) and Sciaphila thaidanica (Petersen et al. 2018), both of which only harbor the two tRNAs. Cryptically, it could not explain why no functional tRNAs was detected in two species of Pilostyles (Bellot and Renner 2015). Therefore, the evolution of the tRNAs in plastomes is cryptic and remains overlooked. Taken together, loss of tRNAs is likely species-/lineage-specific and undergoes varying degrees within heterotrophs.

\section{Unusual retention of genes in $B$. itoana plastome}

Generally, plastomes of heterotrophs which at the fourth stage of degradation begin to shed all coding regions for photosynthetic pathway (Barrett et al. 2014; Graham et al. 2017; Wicke and Naumann 2018). Attractively, petG which encodes a subunit protein of the cytochrome b6/f complex for connection of PSI and PSII (Bock 2007; Wicke et al. 2011), is idiosyncratically retained as a pseudogene in $B$. itoana plastome but is not detected in other heterotrophs at the advanced stage of degradation. One hypothesis suggest that proximity to the essential gene or essential function can help genes escaping from being lost (Lohan and Wolfe 1998). In plastome of B. itoana, petG is proximate to trnW_CCA and accD, two genes could prolong retention in plastome of most heterotrophs. However, petG is totally lost while trnW_CCA and accD are intact in plastomes of $R$. gardneri and S. densiflora (Delannoy et al. 2011; Lam et al. 2015). In addition, Naumann et al (2016) suggested that if plastome harbors pseudogenes, degradation of plastome would be ongoing. Holding six pseudogenes implied that plastome of B.itoana may undergo degradation, and these pseudogenes including petG would be totally deleted.

In heterotrophs, the ribosomal genes begin to be absent from plastomes with varying degrees at the advanced stages (Barrett and Davis 2012; Barrett et al. 2014; Naumann et al. 2016; Wicke et al. 2016; Graham et al. 2017). So far, no study reported functional or physical loss of rpl36 while rpl32, rpl20, rpl22, rpl23 and rpl33 are retained. Interestingly, rpl36 become a pseudogene in B. itoana. Some factors, such as short length, location in conserved operon or difficulty to be replaced by plastid compartments, may contribute to the retention of genes (Lohan and Wolfe 1998; Wicke et al. 2013; Wicke et al. 2016). Commonly, rpl36 is located in

PeerJ reviewing PDF | (2019:03:36139:2:0:NEW 19 Aug 2019) 
276 the intergenic space of rps11 and infA in the most conservative operon including almost all of 277 ribosomal protein-coding genes. Therefore, residing in essential operon and adjacent essential 278 genes could not prevent rpl36 from being pseudogenized in B. itoana plastome. It's plausible to 279 speculate that the function of rpl36 is not essential, or its loss can be compensated by other 280 intracellular genomes in B. itoana plastome (Schelkunov et al. 2015; Cusimano and Wicke 2016; 281 Naumann et al. 2016; Petersen et al. 2018).

282 Among the ribosomal genes, rps15 and rpl32 are the first batch of genes to be absent from 283 reported plastomes at the advanced stage of degradation. However, rps15 and rpl32 are still 284 retained and putatively functional in B. itoana plastome. Previous study suggested that there is a 285 strong positive correlation between the number of putatively functional genes and plastome 286 length among heterotrophic angiosperms (Barrett and Kennedy 2018). To date, the two genes $287 \mathrm{rps} 15$ and rpl32 are only preserved in reported plastomes whose length are longer than $80 \mathrm{~kb}$ 288 only except the Monotropa hypopitys plastome with ca. $40 \mathrm{kbp}$ in length (Ravin et al. 2016). In 289 B. itoana plastome, rps15 and rpl32 are resided in IRs, typically located in SSC. Previous studies 290 proved that two copies of genes in IR offer more opportunities to correct the aberrant mutations 291 (Zhu et al. 2016; Choi et al. 2018). Thus, we could reasonably speculate that IRs may shelter 292 these genes from being deleted in B. itoana plastome.

293 Multiple origination of rearrangements in B. itoana plastome

294 It has been showed that some rearrangements are correlated with intermolecular recombination 295 between distinct tRNA (Hiratsuka et al. 1989; Haberle et al. 2008; Barrett and Kennedy 2018). In 296 plastomes of autotroph $B$. disticha, the cluster in Inv1 is part of the cluster of trnS_UGA - psbZ 297 trnG_GCC - trnfM_CAU - rps 14 - psaB - psaA - ycf3 - trnS_GGA. Thus we postulate that the 298 recombination of trnS_UGA and trnS_GGA may result in the Inv1 of $B$. itoana plastome as the 299 39-kb inversion meditated by a pair of 29-bp inverted repeats located in the trnS_GGA and 300 trnS_GCU in Tylosema fassoglensis plastome (Wang et al. 2018). Previous comparative analysis 301 between plastome of heterotrophic plant Petrosavia stellaris and that of its photosynthetic 302 303 304 305 306 307 relative Japonolirion osense suggested that the rearrangements in the $P$. stellaris plastome are likely associated with transition to heterotrophic way of life (Logacheva et al. 2014). Herein, the accD (Inv2) in the plastome of $B$. itoana is inverted versus that in its photosynthetic relative $B$. disticha. Inversion of accD is not only absent from the plastomes of heterotrophic plants $P$. stellaris and S. densiflora (Logacheva et al. 2014; Lam et al. 2015), but also has occurred independently in many autotrophic plants, e.g., Tylosema esculentum (Fabaceae, KX792933), Passiflora edulis (Passifloraceae, KX290855) and Scaevola taccada (Goodeniaceae, MK397896). In autotrophic plants, the inversion of accD is mostly resulted from the inversion of a block containing accD and other genes. Commonly, accD is located in a block mainly consisting of the photosynthesis related genes, most of which were lost in B. itoana plastome. It needs more studies to hypothesize if gene losses have triggered the inversion along with the shift from autotroph to heterotroph.

In addition, few rearrangements are located in the middle of IRs in plastomes of land plants restricted rare lineages, such as some ferns (Robison et al. 2018), lycophyte (Mower et al. 2019), 
316 Geraniaceae (Weng et al. 2014). Interestingly, four rearrangements consisting of two inversions 317 (Inv3/Inv4) and two translocations (Trans1/Trans2) involved in the IRs and SSC are identified in 318 B. itoana plastome. The two inversions, which are typically located in IRs, are detected across 319 the IRs and SSC. The two translocations (Trans1/Trans2), which are typically located in the 320 junction of IRs and SSC, are translocated into IRs of B. itoana plastome. It was hypothesized 321 that MORFFO (Mobile Open Reading Frames in Fern Organelles) elements are regularly 322 associated with inversions and changes to the inverted repeats (Robison et al. 2018). In our 323 study, the patterns of these rearrangements are analogous to those of rearrangements in several 324 plastomes of ferns (Robison et al. 2018). However, there is no unusual open reading frames (ORF) in the B. itoana plastome, while unusual ORF was identified in the ferns plastomes.

327

328

329

330

331

332

333

334

335

336

337

338

339

340

341

342

343

344

345

346

347

348

349

350

351

352

353

354

355 Hence, we suggest that the four rearrangements in B. itoana plastome could not be consequence of mobile element as in the ferns plastome. Likewise, gene relocation was detected in the plastomes of Oleaceae (Jasminum and Menodora) (Lee et al. 2007) and lycophyte (Mower et al. 2019). Both studies suggested that gene relocation attributes to overlapping inversions rather than the direct transposition or intragenomic translocation. Therefore we speculate that the four rearrangements detected in IRs and SSC of B. itoana plastome may be resulted from multiple expansions and contractions of IRs as in the plastome of Pelargonium hortorum (Chumley et al. 2006) and overlapping inversions as those in Jasminum, Menodora and lycophyte. Firstly, several blocks in SSC (e.g., rpl32, rps15 and ndhD) translocated into the IRs resulting from expansion of IRs in the plastome from the ancestor of $B$. itoana. Then the block including these genes in the four rearrangements of $B$. itoana plastome was inverted, which could result in the translocations (Tans1/Trans2) including rps15, rp132, ndhD, $\operatorname{trnN}$ and ycf1. After that, one inversion may occur between the first exon of ndhB and Tans1/Trans2, and then the first exon of ndhB could be inverted once again. Otherwise, contraction of IRs could explain the translocation and loss of one copy of the block including rps7 and rps12 in Inv3. If intermediate steps are detected in the plastomes of Burmannia lineages as those in lycophyte (Mower et al. 2019), then these could provide a better explanation for that the multiple waves of the IRs and overlapping inversions contribute to the four rearrangements in B. itoana plastome.

\section{Conclusion}

Consistent with the convergent evolution of plants plastomes en route to heterotroph, B. itoana plastome exhibits rampant degradation and distinct rearrangements. Based on models of plastome evolution, B. itoana plastome is at the advanced stages of degradation, and occurrence of six putative pseudogenes suggests that it could undergo further degradation. Unusual retention of genes and diverse rearrangements indicate that complex constraints affect the fate of the plastomes in heterotrophs. Although, heterotrophs share a universal pattern of plastome degradation, the evolution of plastomes from autotrophs to heterotrophs may be species-specific or lineage-specific. In general, our study fills the gap of knowledge about the plastome of heterotrophs in Burmanniaceae, and it would be attractive to investigate plastomic evolution in the genus with more species and more populations within species to characterize the trajectories of plastomic evolution. 
356

357

358

359

360

361

362

363

364

365

366

367

368

369

370

371

372

373

374

375

376

377

378

379

380

381

382

383

384

385

386

387

388

389

390

391

392

393

394

395

\section{References}

Barbrook AC, Howe CJ, Purton S (2006) Why are plastid genomes retained in nonphotosynthetic organisms? Trends in Plant Science 11:101-108

doi:10.1016/j.tplants.2005.12.004

Barrett CF, Davis JI (2012) The plastid genome of the mycoheterotrophic Corallorhiza striata (Orchidaceae) is in the relatively early stages of degradation. American journal of botany 99:1513-1523 doi:10.3732/ajb.1200256

Barrett CF, Freudenstein JV, Li J, Mayfield-Jones DR, Perez L, Pires JC, Santos C (2014) Investigating the path of plastid genome degradation in an early-transitional clade of heterotrophic orchids, and implications for heterotrophic angiosperms. Molecular Biology and Evolution 31:3095-3112 doi:10.1093/molbev/msu252

Barrett CF, Kennedy AH (2018) Plastid genome degradation in the endangered, mycoheterotrophic, North American orchid Hexalectris warnockii. Genome biology and evolution 10:1657-1662 doi:10.1093/gbe/evy107

Bellot S, Renner SS (2015) The plastomes of two species in the endoparasite genus Pilostyles (Apodanthaceae) each retain just five or six possibly functional genes. Genome biology and evolution 8:189-201 doi:10.1093/gbe/evv251

Bock R (2007) Structure, function, and inheritance of plastid genomes. In: Bock R (ed) Cell and Molecular Biology of Plastids, vol 19. Topics in Current Genetics. Springer, pp 29-63. doi:10.1007/4735_2007_0223

Choi KS, Jeong KS, Ha Y-H, Choi K (2018) Complete chloroplast genome sequences of Clematis IR expansion and relative rates of synonymous substitutions. doi:10.20944/preprints201804. 0106.v1

Chumley TW, Palmer JD, Mower JP, Fourcade HM, Calie PJ, Boore JL, and Jansen RK. 2006. The complete chloroplast genome sequence of Pelargonium $\mathrm{x}$ hortorum: organization and evolution of the largest and most highly rearranged chloroplast genome of land plants. Molecular Biology and Evolution 23:2175-2190. 10.1093/molbev/ms1089

Cusimano N, Wicke S (2016) Massive intracellular gene transfer during plastid genome reduction in nongreen Orobanchaceae. New Phytologist 210:680-693 doi:10.1111/nph. 13784

Darling ACE, Mau B, Blattner FR, Perna NT (2004) Mauve: Multiple alignment of conserved genomic sequence with rearrangements. Genome Research 14:1394-1403 doi:10.1101/gr.2289704

Delannoy E, Fujii S, Colas des Francs-Small C, Brundrett M, Small I (2011) Rampant gene loss in the underground orchid Rhizanthella gardneri highlights evolutionary constraints on plastid genomes. Molecular Biology and Evolution 28:2077-2086 doi:10.1093/molbev/msr028

Feng YL, Wicke S, Li JW, Han Y, Lin CS, Li DZ, Zhou TT, Huang WC, Huang LQ, and Jin XH. (2016) Lineage-Specific reductions of plastid genomes in an orchid tribe with partially and fully mycoheterotrophic species. Genome biology and evolution 8:2164-2175 
396

397

398

399

400

401

402

403

404

405

406

407

408

409

410

411

412

413

414

415

416

417

418

419

420

421

422

423

424

425

426

427

428

429

430

431

432

433

434

435

doi:10.1093/gbe/evw144

Figueroa-Martinez F, Nedelcu AM, Smith DR, Reyes-Prieto A (2017) The plastid genome of Polytoma uvella is the largest known among colorless algae and plants and reflects contrasting evolutionary paths to nonphotosynthetic lifestyles. Plant physiology 173:932943 doi:10.1104/pp.16.01628

Gould SB, Waller RF, McFadden GI (2008) Plastid evolution. Annual review of plant biology 59:491-517 doi: 10.1146/annurev.arplant.59.032607.092915

Graham SW, Lam VK, Merckx VS (2017) Plastomes on the edge: the evolutionary breakdown of mycoheterotroph plastid genomes. New phytologist 214:48-55 doi:10.1111/nph.14398

Haberle RC, Fourcade HM, Boore JL, Jansen RK (2008) Extensive rearrangements in the chloroplast genome of Trachelium caeruleum are associated with repeats and tRNA genes. Journal of Molecular Evolution 66:350-361 doi:10.1007/s00239-008-9086-4

Hiratsuka J, Shimada H, Whittier R, Ishibashi T, Sakamoto M, Mori M, Kondo C, Honji Y, Sun C-R, Meng B-Y, Li Y-Q, Kanno A, Nishizawa Y, Hirai A, Shinozaki K, Sugiura M. (1989) The complete sequence of the rice (Oryza sativa) chloroplast genome: Intermolecular recombination between distinct tRNA genes accounts for a major plastid DNA inversion during the evolution of the cereals. Molecular General Genetics 217:185-194 doi: $10.1007 / \mathrm{bf} 02464880$

Jonker FP (1938) A monograph of the Burmanniaceae. Kemink en zoon,

Kearse M, Moir R, Wilson A, Stones-Havas S, Cheung M, Sturrock S, Buxton S, Cooper A, Markowitz S, and Duran C. (2012) Geneious Basic: An integrated and extendable desktop software platform for the organization and analysis of sequence data. Bioinformatics 28:1647 doi: 10.1093/bioinformatics/bts199

Kim JS, Kim HT, Kim J-H (2014) The largest plastid genome of monocots: a novel genome type containing AT residue repeats in the slipper orchid Cypripedium japonicum. Plant Molecular Biology Reporter 33:1210-1220 doi:10.1007/s11105-014-0833-y

Ku C, Nelson-Sathi S, Roettger M, Garg S, Hazkani-Covo E, Martin WF (2015) Endosymbiotic gene transfer from prokaryotic pangenomes: Inherited chimerism in eukaryotes. Proceedings of the National Academy of Sciences of the United States of America 112:10139-10146 doi:10.1073/pnas.1421385112

Lam VK, Soto Gomez M, Graham SW (2015) The highly reduced plastome of mycoheterotrophic Sciaphila (Triuridaceae) is colinear with its green relatives and is under strong purifying selection. Genome biology and evolution 7:2220-2236 doi:10.1093/gbe/evv134

Liere K, Link G (1995) RNA-binding activity of the matK protein encodecd by the chloroplast trnk intron from mustard (Sinapis alba L.). Nucleic Acids Research 23:917-921 doi: 10.1093/nar/23.6.917

Lim GS, Barrett CF, Pang CC, Davis JI (2016) Drastic reduction of plastome size in the mycoheterotrophic Thismia tentaculata relative to that of its autotrophic relative Tacca chantrieri. American journal of botany 103:1129-1137 doi:10.3732/ajb.1600042 
436 Lee HL, Jansen RK, Chumley TW, Kim KJ. 2007. Gene relocations within chloroplast genomes

437

438

439

440

441

442

443

444

445

446

447

448

449

450

451

452

453

454

455

456

457

458

459

460

461

462

463

464

465

466

467

468

469

470

471

472

473

474

475 of Jasminum and Menodora (Oleaceae) are due to multiple, overlapping inversions. Molecular Biology and Evolution 24:1161-1180. 10.1093/molbev/msm036

Logacheva MD, Schelkunov MI, Nuraliev MS, Samigullin TH, Penin AA (2014) The plastid genome of mycoheterotrophic monocot Petrosavia stellaris exhibits both gene losses and multiple rearrangements. Genome biology and evolution 6:238-246 doi:10.1093/gbe/evu001

Lohan AJ, Wolfe KH (1998) A subset of conserved tRNA genes in plastid DNA of nongreen plants. Genetics 150:425-433 doi: 10.1046/j.1365-2443.1998.00217.x

Lohse M, Drechsel O, Bock R (2007) OrganellarGenomeDRAW (OGDRAW): a tool for the easy generation of high-quality custom graphical maps of plastid and mitochondrial genomes. Current Genetics 52:267-274 doi: 10.1007/s00294-007-0161-y

Ma L, Ma P, Li D (2018) The first complete plastid genome of Burmannia disticha L. from the mycoheterotrophic monocot family Burmanniaceae. Plant Diversity 40:232-237 doi:10.1016/j.pld.2018.07.004

Martin W, Herrmann RG (1998) Gene transfer from organelles to the nucleus: How much, what happens, and why? Plant physiology 118:9-17 doi:10.1104/pp.118.1.9

McNeal JR, Kuehl JV, Boore JL, Leebens-Mack J, dePamphilis CW (2009) Parallel loss of plastid introns and their maturase in the genus Cuscuta. PLoS One 4:e5982 doi:10.1371/journal.pone.0005982

Merckx VSFT, Gomes SIF, Wapstra M, Hunt C, Steenbeeke G, Mennes CB, Walsh N, Smissen R, Hsieh T-H, Smets EF, Bidartondo MI. 2017. The biogeographical history of the interaction between mycoheterotrophic Thismia (Thismiaceae) plants and mycorrhizal Rhizophagus (Glomeraceae) fungi. Journal of Biogeography 44:1869-1879. 10.1111/jbi.12994

Mohr G, Lambowitz AM (2003) Putative proteins related to group II intron reverse transcriptase/maturases are encoded by nuclear genes in higher plants. Nucleic Acids Research 31:647-652 doi:10.1093/nar/gkg153

Molina J, Hazzouri KM, Nickrent D, Geisler M, Meyer RS, Pentony MM, Flowers JM, Pelser P, Barcelona J, Inovejas SA, Uy I, Yuan W, Wilkins O, Michel CI, Locklear S, Concepcion GP, and Purugganan MD. 2014. Possible loss of the chloroplast genome in the parasitic flowering plant Rafflesia lagascae (Rafflesiaceae). Molecular Biology and Evolution 31:793-803 doi:10.1093/molbev/msu051

Mower JP, Ma PF, Grewe F, Taylor A, Michael TP, VanBuren R, and Qiu YL. 2019. Lycophyte plastid genomics: extreme variation in $\mathrm{GC}$, gene and intron content and multiple inversions between a direct and inverted orientation of the rRNA repeat. New phytologis 222:10611075. 10.1111/nph.15650

Naumann J, Der JP, Wafula EK, Jones SS, Wagner ST, Honaas LA, Ralph PE, Bolin JF, Maass E, Neinhuis C, Wanke S, dePamphilis CW. (2016) Detecting and characterizing the highly divergent plastid genome of the nonphotosynthetic parasitic plant Hydnora visseri 
476

477

478

479

480

481

482

483

484

485

486

487

488

489

490

491

492

493

494

495

496

497

498

499

500

501

502

503

504

505

506

507

508

509

510

511

512

513

514

515

(Hydnoraceae). Genome biology and evolution 8:345-363 doi:10.1093/gbe/evv256

Petersen G, Zervas A, Pedersen HAE, Seberg O (2018) Genome Reports: Contracted genes and dwarfed plastome in Mycoheterotrophic Sciaphila thaidanica (Triuridaceae, Pandanales). Genome biology and evolution 10:976-981 doi:10.1093/gbe/evy064

Robison TA, Grusz AL, Wolf PG, Mower JP, Fauskee BD, Sosa K, Schuettpelz E (2018) Mobile elements shape plastome evolution in ferns. Genome biology and evolution 10:2558-2571 doi:10.1093/gbe/evy189

Roquet C, Coissac E, Cruaud C, Boleda M, Boyer F, Alberti A, Gielly L, Taberlet P, Thuiller W, Van Es J, Lavergne S. 2016. Understanding the evolution of holoparasitic plants: the complete plastid genome of the holoparasite Cytinus hypocistis (Cytinaceae). Annals of Botany 118:885-896. doi: 10.1093/aob/mcw135

Schattner P, Brooks AN, Lowe TM (2005) The tRNAscan-SE, snoscan and snoGPS web servers for the detection of tRNAs and snoRNAs. Nucleic Acids Research 33: W686 doi: 10.1093/nar/gki366

Schelkunov MI, Shtratnikova VY, Nuraliev MS, Selosse MA, Penin AA, Logacheva MD (2015) Exploring the limits for reduction of plastid genomes: a case study of the mycoheterotrophic orchids Epipogium aphyllum and Epipogium roseum. Genome biology and evolution 7:1179-1191 doi:10.1093/gbe/evv019

Schneider AC, Braukmann T, Banerjee A, Stefanovic S (2018) Convergent plastome evolution and gene loss in holoparasitic Lennoaceae (Boraginales). Genome biology and evolution doi:10.1093/gbe/evy190

Smith DR, Lee RW (2014) A Plastid without a Genome: Evidence from the Nonphotosynthetic Green Algal Genus Polytomella. Plant physiology 164:1812-1819 doi:10.1104/pp.113.233718

Tillich M, Lehwark P, Pellizzer T, Ulbricht-Jones ES, Fischer A, Bock R, Greiner S (2017) GeSeq - versatile and accurate annotation of organelle genomes. Nucleic Acids Research 45 doi: 10.1093/nar/gkx391

Wang YH, Wicke S, Wang H, Jin JJ, Chen SY, Zhang SD, Li DZ, and Yi TS. 2018. Plastid genome evolution in the early-diverging legume subfamily Cercidoideae (Fabaceae). Frontiers in Plant Science 9:138. 10.3389/fpls.2018.00138

Weng ML, Blazier JC, Govindu M, Jansen RK (2014) Reconstruction of the ancestral plastid genome in Geraniaceae reveals a correlation between genome rearrangements, repeats, and nucleotide substitution rates. Molecular Biology and Evolution 31:645-659 doi:10.1093/molbev/mst257

Wicke S, Muller KF, de Pamphilis CW, Quandt D, Wickett NJ, Zhang Y, Renner SS, and Schneeweiss GM. 2013. Mechanisms of functional and physical genome reduction in photosynthetic and nonphotosynthetic parasitic plants of the broomrape family. Plant Cell 25:3711-3725. doi:10.1105/tpc.113.113373

Wicke S, Muller KF, dePamphilis CW, Quandt D, Bellot S, Schneeweiss GM (2016) Mechanistic model of evolutionary rate variation en route to a nonphotosynthetic lifestyle 
516 in plants. Proceedings of the National Academy of Sciences of the United States of

$517 \quad$ America 113:9045-9050 doi:10.1073/pnas.1607576113

518 Wicke S, Naumann J (2018) Molecular evolution of plastid genomes in parasitic flowering

519 plants. In: Chaw S-M, Jansen RK (eds) Advances in Botanical Research, vol 85. Academic

$520 \quad$ Press, pp 315-347. doi: 10.1016/bs.abr.2017.11.014

521 Wicke S, Schneeweiss GM, dePamphilis CW, Muller KF, Quandt D (2011) The evolution of the

522 plastid chromosome in land plants: gene content, gene order, gene function. Plant

523 Molecular Biology 76:273-297 doi:10.1007/s11103-011-9762-4

524 Wu D, Zhang D, Saunders. RMK (2010) Burmanniaceae Wu, ZY, Raven, PH and Hong, DY

525 (Eds), Flora of China 23:121-125

526 Zhang D (1999) Systematics of Burmannia L. (Burmanniaceae) in the old world Hku Theses

$527 \quad$ Online

528 Zhu A, Guo W, Gupta S, Fan W, Mower JP (2016) Evolutionary dynamics of the plastid inverted

529 repeat: the effects of expansion, contraction, and loss on substitution rates. New phytologist

$530 \quad 209: 1747-1756$ doi:10.1111/nph.13743

531 Zoschke R, Nakamura M, Liere K, Sugiura M, Boerner T, Schmitz-Linneweber C (2010) An

532 organellar maturase associates with multiple group II introns. Proceedings of the National

533 Academy of Sciences of the United States of America 107:3245-3250

534 doi:10.1073/pnas.0909400107 
Figure 1

Burmannia itoana in flower, Long Men Park, Guang Dong, China. 


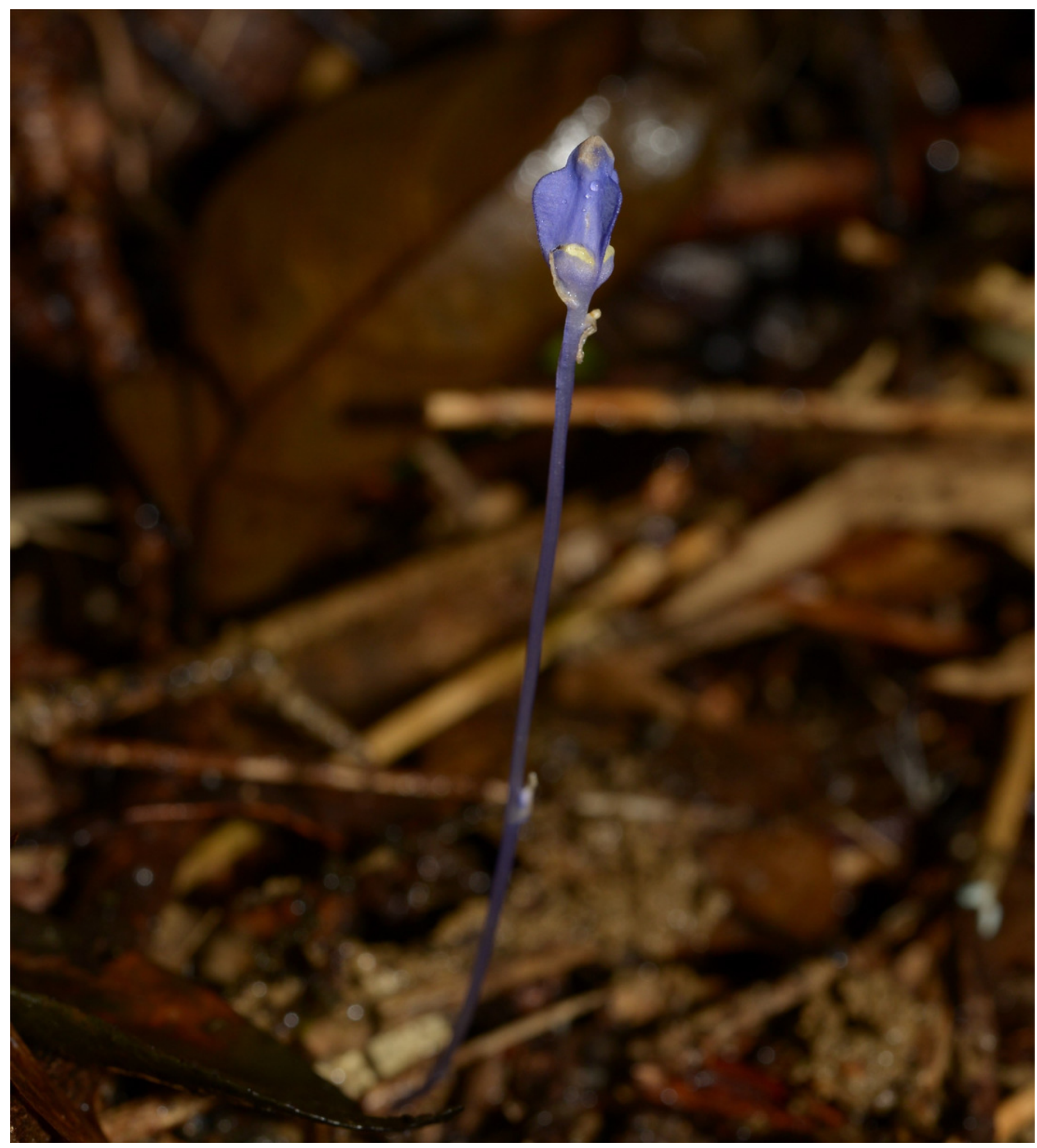


Figure 2

Plastome structure of five species in Dioscoreales.

All genes are colored according to functional complexes. Genes shown left the line are transcribed counterclockwise, those right the line are transcribed clockwise. The light red blocks show inversions and light blue blocks show insertions. Genes containing intron(s) are marked with the symbol *. Pseudogenes and truncated genes are marked with " $\&$ " and "\#", respectively. The inverted repeats (IRs) are shown with bold lines. 


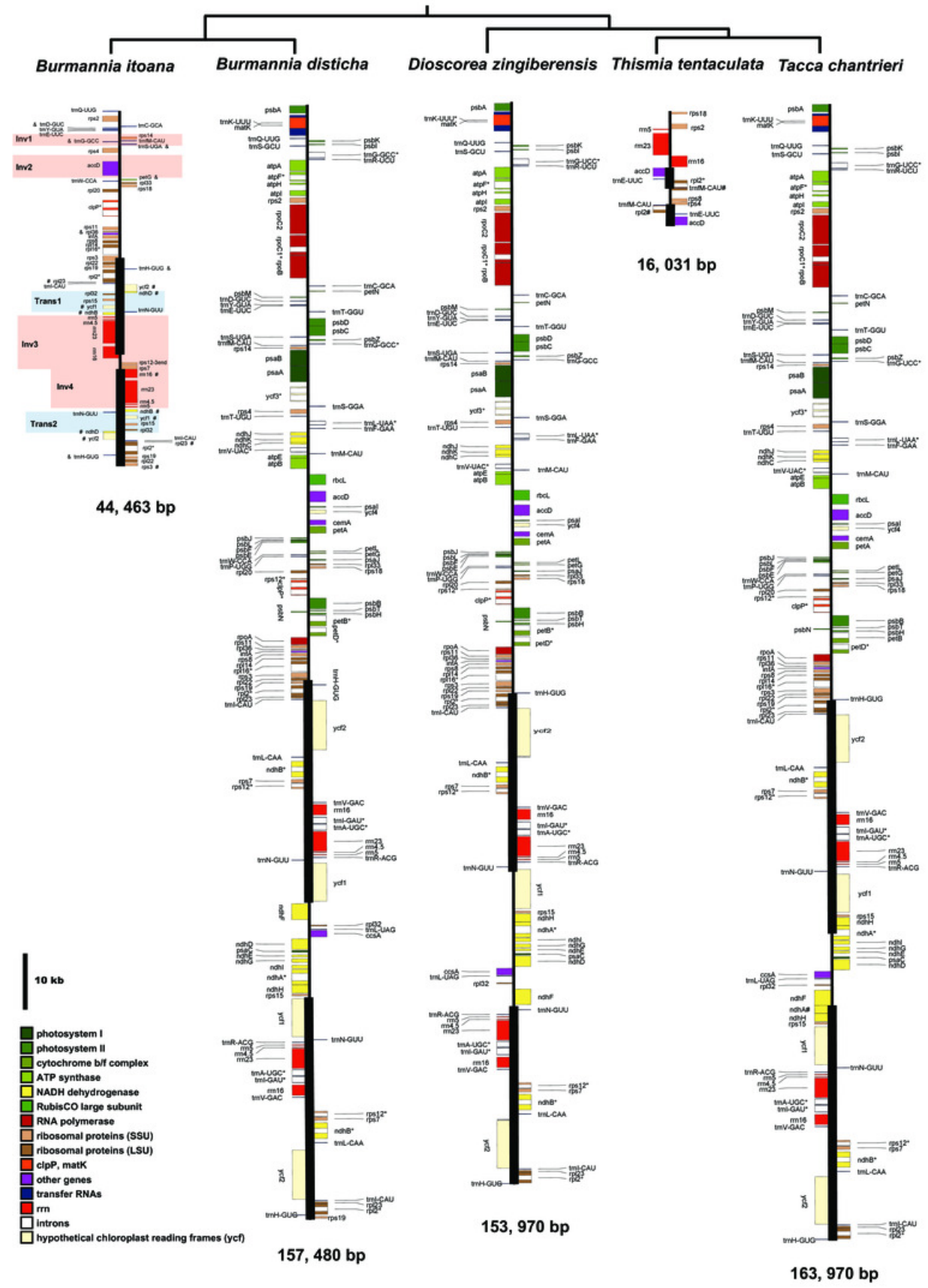




\section{Figure 3}

Heat map showing gene contents in five species in Dioscoreales.

Genes in blue are retained and presumed fully functional; those in red are absent and those in yellow are putative pseudogenes. Gene names of photosynthesis related genes, genetic apparatus genes and other genes are in green, black, and red, respectively. 


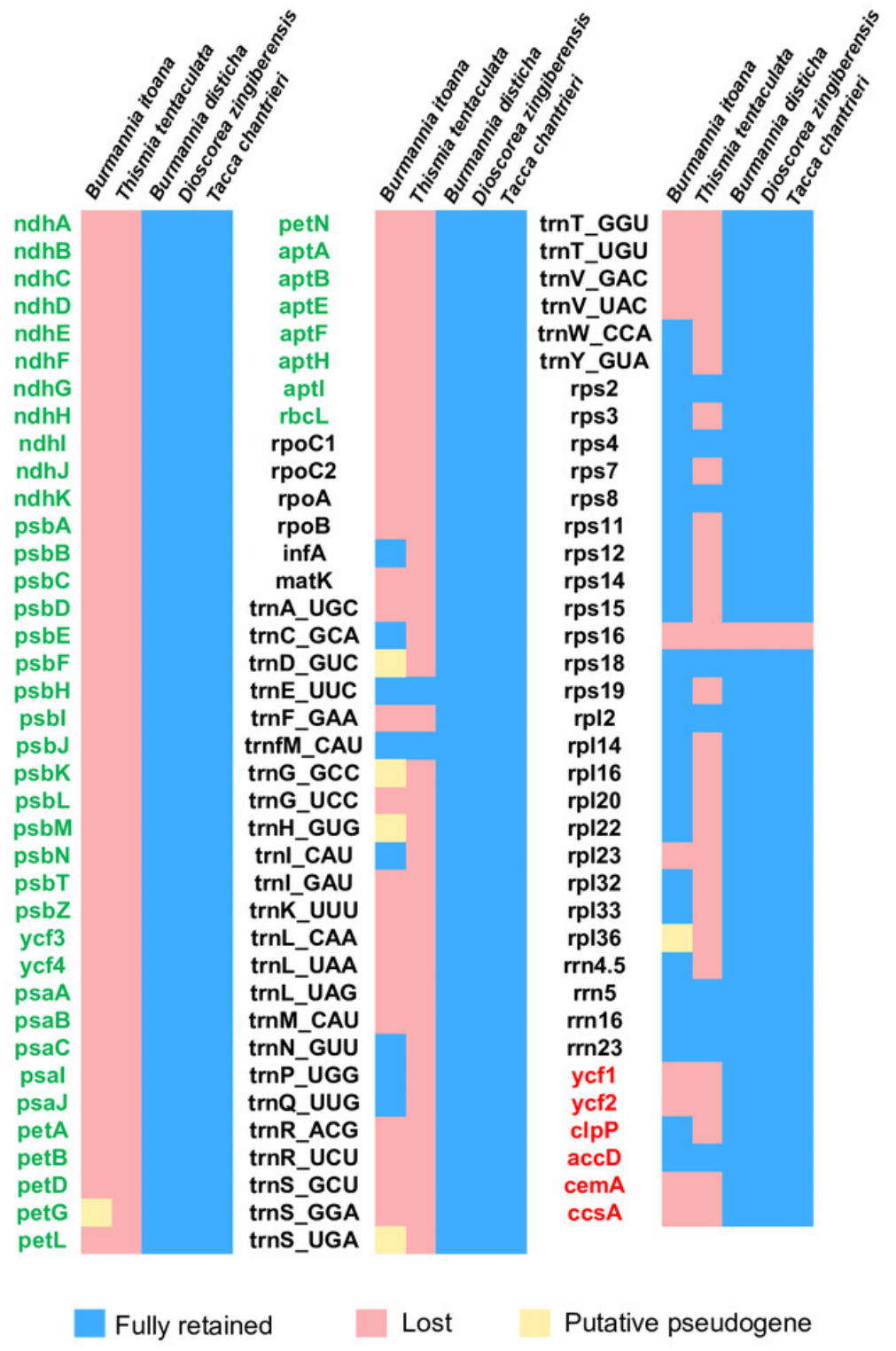


Figure 4

Synteny of plastomes from five species .

The dashed lines and olidlines illustrate rearranged gene blocks and collinear between the two plastomes, respectively.

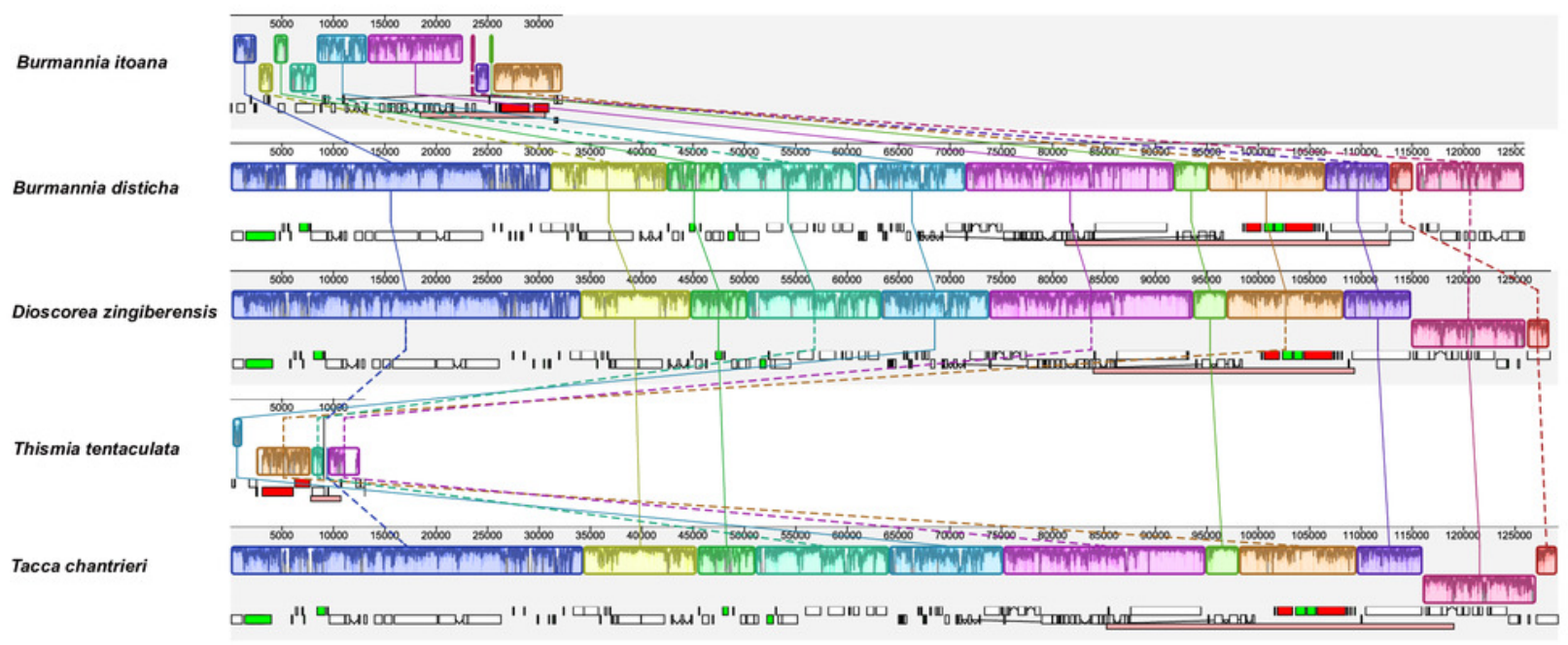




\section{Table 1 (on next page)}

General information of plastomes from five species in Dioscoreales. 


\begin{tabular}{cccccccc}
\hline \multirow{2}{*}{ Taxon } & \multicolumn{4}{c}{ Length (bp) /GC-content (\%) } & \multicolumn{3}{c}{ Gene number } \\
\cline { 2 - 8 } & Total & IR & LSC & SSC & rRNAs & tRNAs & Protein coding \\
\hline Burmannia itoana & $44463 / 32.0$ & $12174 / 37.8$ & $18441 / 24.5$ & $1674 / 32.3$ & 4 & 12 & 23 \\
Burmannia disticha & $157480 / 34.9$ & $31616 / 39.5$ & $81231 / 32.3$ & $13017 / 28.8$ & 4 & 30 & 78 \\
Dioscorea zingiberensis & $153970 / 37.2$ & $25491 / 43.0$ & $83950 / 35.1$ & $19038 / 31.2$ & 4 & 30 & 78 \\
Tacca chantrieri & $163007 / 36.7$ & $33837 / 40.3$ & $85241 / 34.7$ & $10092 / 30.6$ & 4 & 30 & 78 \\
Thismia tentaculata & $16031 / 27.2$ & $2948 / 30.0$ & $7799 / 29.1$ & $2336 / 13.7$ & 3 & 2 & 7 \\
\hline
\end{tabular}

